\title{
Testosterone deficiency and apathy in Parkinson's disease: a pilot study
}

\author{
R E Ready, J Friedman, J Grace, H Fernandez
}

See Editorial Commentary, p1232

J Neurol Neurosurg Psychiatry 2004;75:1323-1326. doi: 10.1136/jnnp.2003.032284

Background: Low testosterone in men with Parkinson's disease may be associated with non-motor symptoms of the disease, such as apathy.

Objective: To determine the association between free serum testosterone level and apathy in elderly men with Parkinson's disease.

Methods: Consecutive non-demented patients $(n=49)$ and knowledgeable informants $(n=40)$ participated in the study. Patients and informants reported on apathy using the Frontal Systems Behavior Scale and two visual analogue scales. Patients also provided self reported symptoms of depression on the Beck depression inventory-ll. Blood samples were drawn at the time of assessment to determine testosterone levels.

Results: A low total testosterone concentration was found in $46.9 \%$ of the patients, defined as $\leqslant 325 \mathrm{ng} / \mathrm{dl}$. Free testosterone was significantly correlated with both patient reported and informant reported apathy, independent of disease severity.

Conclusions: Apathy is common in Parkinson's disease and is inversely correlated with free testosterone. Testosterone replacement therapy could be considered as a potential treatment for apathy in some men with Parkinson's disease. More research is needed to replicate these findings and to investigate the response to treatment.

S ome of the non-motor symptoms of Parkinson's disease may be associated with testosterone deficiency. ${ }^{12}$ Low testosterone in male patients is associated with decreased libido, erectile dysfunction, fatigue, decreased enjoyment in life, mood changes, and deterioration in work performance. ${ }^{2}$ Many of these symptoms are responsive to treatment, making their identification in individual patients with Parkinson's disease potentially important. ${ }^{1-3}$

We hypothesised that testosterone deficiency in this disease is directly related to increased apathy, a problem not yet linked to low testosterone levels. Frontal systems dysfunction is strongly implicated in Parkinson's disease, ${ }^{4}$ and apathy is linked to frontal systems dysfunction..$^{5-7}$ Thus one mechanism for the apathy that is a common finding in patients with Parkinson's disease ${ }^{6-10}$ might be frontal systems dysfunction. Testosterone deficiency in male patients could exacerbate frontal systems impairment because testosterone may subserve frontal systems. For example, there is evidence from experimental animal research ${ }^{11}$ and human studies ${ }^{12}$ that testosterone administration in male subjects facilitates functions that are subserved by the prefrontal cortex, such as working memory.

We report our findings of an investigation of the association between testosterone levels and apathy in a sample of non-demented male patients with Parkinson's disease.

\section{METHODS \\ Participants}

Male patients diagnosed with idiopathic Parkinson's disease $(\mathrm{n}=60)$ were invited to participate in the study, and 49 completed the protocol. Parkinson's disease was diagnosed by movement disorder specialists (JF, HF) in a movement disorder clinic by the presence of three of the four cardinal criteria: tremor at rest, bradykinesia, rigidity, and postural instability. Patients did not have atypical features, other explanations for parkinsonism, or any other neurological disorder. Patients were not recruited if they met DMS-IV diagnostic criteria for dementia. ${ }^{13}$ A subset of patients had an informant participate in the study. Informants knew the patients very well and had daily contact with them.

\section{Measures \\ Frontal Systems Behavior Scale}

The Frontal Systems Behavior Scale (FrSBe) $)^{14}$ was completed by patients and informants. The scale measures initiation, psychomotor retardation, spontaneity, drive, persistence, loss of energy and interest, lack of concern, and blunted affective expression. It has strong internal consistency reliability and convergent validity. The scale is not significantly correlated with depressed mood. ${ }^{15}$ Higher scores indicate greater apathy.

Apathy visual analogue scales

Two visual analogue scales (VAS) for apathy were created and completed by patients and informants. Both scales had vertical $100 \mathrm{~mm}$ lines with verbal anchors on each end: (1) Extremely interested versus Not interested; (2) Feel things deeply versus Without feeling. Lower scores on the scales indicate greater apathy.

The Beck depression inventory, second edition The Beck depression inventory, second edition (BDI-II ${ }^{16}$ is a 21 item self report inventory that measures depressive symptoms over the previous two weeks. Higher scores reflect greater symptoms of depression. The BDI-II has strong internal consistency, reliability, and convergent validity with other measures of depressive symptoms.

\section{Testosterone laboratory tests}

Free and total serum testosterone levels were measured. Laboratory tests were done by Quest Diagnostics. ${ }^{17}$

Abbreviations: BDI-II, Beck depression inventory, second edition; DMSIV, Diagnostic and Statistical Manual of Mental Disorders, 4th edition; $\mathrm{FrSBe}$, trontal systems behavioural apathy rating scale; VAS, visual analogue scale 


\section{Procedure}

All consecutive eligible outpatients with Parkinson's disease seen in the neurology department of the Memorial Hospital of Rhode Island (MHRI) were invited to participate. The protocol was approved by the institutional review board of MHRI. After informed consent had been obtained, patients completed the self report questionnaires. A blood sample was drawn to assess testosterone levels. Patients were also asked permission to have an informant participate in the study. Informants completed questionnaires based on their interactions with patients in a home setting. Eight patients did not have an informant who was able to participate. For the other 41 patients, questionnaires were sent to the informants' homes.

\section{RESULTS}

\section{Patient and informant characteristics}

Descriptive statistics for patient and caregiver demographic factors are given in table 1. The mean (SD) modified Hoehn and Yahr rating ${ }^{18}$ for patients was 2.5 (1.0). Questionnaires were returned from 40 of the 41 informants, a response rate of $98 \%$. Most informants were spouses $(92.5 \%)$ and lived with the patient (95\%). Informants had been acquainted with patients for an average of 41.1 (16.4) years.

\section{Descriptive statistics for apathy and depression measures}

Descriptive statistics for the testosterone, apathy, and depression measures are included in table 1. The mean scores for patient report on the FrSBe were greater than mean scores for healthy men of similar age and educational background. ${ }^{14}$ The mean score for the BDI-II was in the range of minimal depressive symptoms, and the majority of scores $(62.5 \%)$ were in the minimal range. ${ }^{16}$ Fourteen patients reported mild depressive symptoms (29.2\%), three had moderate symptoms $(6.3 \%)$, and one reported severe symptoms $(2.1 \%)$.

\begin{tabular}{|c|c|c|c|}
\hline & Mean (SD) & Min & $\operatorname{Max}$ \\
\hline \multicolumn{4}{|l|}{ Patients $(n=49)$} \\
\hline Age (y) & $68.9(10.9)$ & 42 & 87 \\
\hline Education (y) & $15.2(3.0)$ & 9 & 20 \\
\hline Hoehn \& Yahr rating* & $2.5(1.0)$ & 0 & 5 \\
\hline \multicolumn{4}{|l|}{ Informants $(n=40)$} \\
\hline Age $(y)$ & $65.0(11.5)$ & 43 & 85 \\
\hline Education (y) & $14.1(2.5)$ & 9 & 20 \\
\hline Relationship length $(y)$ & $41.1(16.4)$ & 6 & 77 \\
\hline \multicolumn{4}{|l|}{ Testosterone (ng/dl) } \\
\hline Total & $343.8(120.8)$ & 125.0 & 714.0 \\
\hline Free & $58.7(21.9)$ & 14.2 & 119.8 \\
\hline \multicolumn{4}{|l|}{ Patient reported measures } \\
\hline $\mathrm{FrSBe}$ & $27.2(7.2)$ & 12 & 44 \\
\hline VAS "Interested" & $66.7(22.6)$ & 8 & 97 \\
\hline VAS "Feel deeply" & $75.3(19.8)$ & 7 & 100 \\
\hline BDI-II & $11.6(6.3)$ & 1 & 31 \\
\hline \multicolumn{4}{|c|}{ Informant reported measures } \\
\hline $\mathrm{FrSBe}$ & $29.1(9.2)$ & 12 & 50 \\
\hline VAS "Interested" & $61.9(27.6)$ & 7 & 100 \\
\hline VAS "Feel deeply" & $63.3(28.0)$ & 6 & 100 \\
\hline
\end{tabular}

*Hoehn \& Yahr ratings are Parkinson's disease severity ratings (scale range 0 to 5 ).

BDI-II, Beck depression inventory, 2nd ed; FrSBe, Frontal Systems Behavior Scale; Max, maximum; Min, minimum; VAS, visual analogue scale; $y$, years.
Analyses indicated that the self reported apathy on the FrSBe scale was only moderately, yet significantly, correlated with BDI-II scores $(r=0.29, \mathrm{p}<0.05)$. The magnitude of this correlation indicates that only $9 \%$ of the variance of these two measures is shared; thus the scales were largely measuring unique constructs. Informant reported apathy was not significantly correlated with the BDI-II $(r=0.05)$.

\section{Testosterone concentrations}

Both free and total testosterone concentrations were measured. Low total testosterone was defined as $\leqslant 325 \mathrm{ng} / \mathrm{dl}$, as in previous studies with Parkinson's disease samples. ${ }^{219}$ Using this cut off, $46.9 \%$ of the sample had a low total testosterone $(\mathrm{n}=23)$.

Free and total testosterone concentrations were strongly and significantly correlated $(r=0.86, \mathrm{p}<0.001)$. As free and total testosterone levels were highly correlated, we used only free testosterone in all analyses.

\section{Correlations between testosterone concentrations, apathy, and depression}

Both patient and informant reports on the FrSBe apathy scale correlated significantly $(\mathrm{p}<0.05)$ and inversely with free testosterone (Pearson $r=-0.31$ for patients, $r=-0.37$ for informants). These relations are illustrated in fig 1 . Free testosterone concentrations were not significantly correlated with the BDI-II or VAS scales.

The correlations between FrSBe apathy and free testosterone levels were recalculated, controlling for disease severity as measured by the modified Hoehn \& Yahr ratings. Results indicated that the magnitude of the association between patient reported FrSBe apathy and total testosterone was unchanged and still significant $(r=-0.33, \mathrm{p}<0.05)$. The association with informant reported FrSBe apathy was also unchanged and remained statistically significant $(r=-0.35$, $\mathrm{p}<0.05$ ).

Three items on the BDI-II inquire about fatigue, low energy, and loss of interest in sex. These items were analysed separately to determine their correlation with free

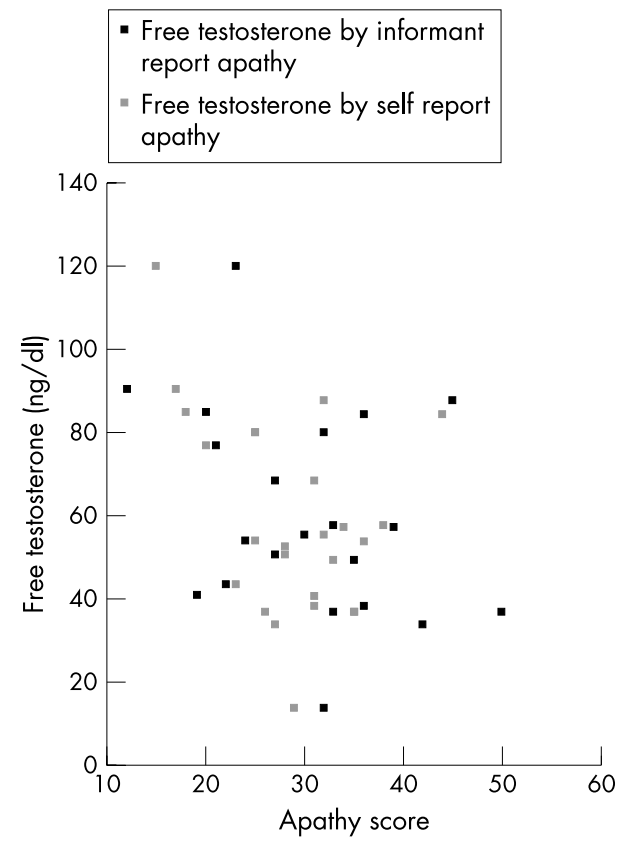

Figure 1 Scatterplot of free testosterone and Frontal Systems Behavior Scale (FrSBe) apathy scores. 
testosterone. The correlations between free testosterone and "fatigue" and "loss of interest in sex" were not statistically significant. However, "low energy" was associated with free testosterone $(r=-0.40, \mathrm{p}<0.05)$.

\section{DISCUSSION}

This pilot study, although small, reveals a potentially important association between testosterone deficiency and apathy. While these results will need to be replicated with prospective epidemiological and treatment studies, they may provide some insight for the clinician encountering the male patient with Parkinson's disease who has testosterone deficiency. Our study confirms the previous observations that testosterone deficiency is not associated with depression per $\mathrm{se}^{20}{ }^{20}$ but may be associated with some depressive features, such as low energy. Clinically, the physician treating testosterone deficiency is often faced with a patient who has "lost their get up and go," or who complains of "feeling out of gas." These are symptoms of apathy that may be associated with testosterone deficiency in some male patients with Parkinson's disease.

Results of this pilot study indicate that patient and informant reports of greater apathy in non-demented male patients are significantly associated with lower levels of free testosterone. Furthermore, the associations were independent of disease severity. Overall, $47 \%$ of the sample had low total testosterone levels, a rate that is somewhat higher than in a previous study where 35\% of patients with Parkinson's disease had low testosterone. ${ }^{1}$

Several measures of apathy were used in the current study, including full length behaviour rating scales and brief visual analogue scales. Significant associations were found only for the longer rating scales. Thus the use of brief instruments that only tap one isolated aspect of the apathy syndrome might not be sufficient to study testosterone deficiency and apathy in Parkinson's disease. Future investigators should carefully select comprehensive and well validated measures of apathy when studying associations with testosterone deficiency.

There are several methodological limitations to the current study. Blood samples were not uniformly taken before 10.00 hours, increasing the likelihood of false positives. ${ }^{19}$ This methodological issue will need to be addressed in future studies. Additionally, participants in this pilot study were not carefully screened for current drug treatment, tobacco and alcohol use, and co-morbid chronic illness, all of which may affect hormone levels. ${ }^{19}$ The limitations regarding interpretation of "normal testosterone" have been addressed by previous investigators. ${ }^{31921}$ We recognise that the lack of a uniform definition of testosterone deficiency makes interpretation of results difficult, despite the data provided by the Baltimore longitudinal study on aging. Another limitation of this study was that we relied on patient self report of symptoms of depression. Future studies should include informant or clinician evaluations of depression as well. In the light of these limitations, the results of this study should be regarded as preliminary and will need confirmation. The other important issue not addressed by the study was whether apathy will be responsive to replacement therapy, and which patients should be candidates. These important questions remain for future studies.

\section{Apathy and testosterone deficiency in Parkinson's disease}

Apathy is associated with frontal systems dysfunction. ${ }^{5-7}$ Frontal systems dysfunction is also strongly implicated in Parkinson's disease. ${ }^{4}$ Thus patients with Parkinson's disease are vulnerable to the apathy syndrome, which is common in this condition ${ }^{6-9}$ because of the frontal systems dysfunction.
Testosterone deficiency in male patients may exacerbate frontal systems dysfunction. For example, testosterone administration in men facilitates functions that are subserved by the prefrontal cortex, such as working memory. ${ }^{11} 12$

Thus testosterone replacement in male patients with Parkinson's disease may be a beneficial treatment as it could facilitate frontal system functions. Results from a recent brain imaging study support this hypothesis, indicating that testosterone replacement therapy in hypogonadal men enhanced cerebral perfusion in midbrain and frontal regions, ${ }^{22}$ which are two regions that are centrally involved in the neuropathology of Parkinson's disease. ${ }^{4}$

If future studies corroborate our findings that testosterone deficiency is associated with symptoms of apathy, then testosterone replacement therapy should be considered as a potential treatment for apathy in men with Parkinson's disease. Two reports involving small samples of patients $(\mathrm{n}=5-10)$ with low testosterone levels who were treated with testosterone replacement therapy reported improvements in mood, energy, libido, and strength, ${ }^{12}$ but apathy was not measured in those studies.

\section{Conclusions}

Our results indicate that low testosterone levels in elderly men diagnosed with Parkinson's disease are associated with symptoms of apathy. These results will need to be replicated. The potential benefits for testosterone replacement therapy for the non-motor aspects of Parkinson's disease ${ }^{2}$ have been preliminarily explored; however, further study is required to determine whether apathy is responsive to treatment. The treatment of apathy in Parkinson's disease with testosterone replacement therapy will require a prospective placebo controlled study. ${ }^{23}$ Practitioners should be cautioned against treating testosterone deficiency in Parkinson's disease until appropriate studies have been completed.

\section{ACKNOWLEDGEMENTS}

We would like to extend our thanks and appreciation to Michael Okun MD for review of an earlier draft of this manuscript. The project was supported by a National Institutes of Health-National Institute of Aging postdoctoral fellowship, 1F32AG20008-01 (RER).

\section{Authors' affiliations}

R E Ready, J Grace, Department of Psychiatry and Human Behavior, Brown Medical School, University of Pittsburgh, Pittsburgh, Pennsylvania, USA

J Friedman, H Fernandez, Department of Clinical Neurosciences, Brown Medical School

Competing interests: none declared

Correspondence to: Dr Rebecca E Ready, The University of Pittsburgh, 121 University Place, Room 513, Pittsburgh, PA 15260, USA; rready@ ucsur.pitt.edu

Received 11 November 2003

Revised 18 February 2004

Accepted 9 April 2004

\section{REFERENCES}

1 Okun MS, McDonald WM, DeLong MR. Refractory nonmotor symptoms in male patients with Parkinson disease due to testosterone deficiency. Arch Neurol 2002;59:807-11.

2 Okun MS, Walter BL, McDonald WM, et al. Beneficial effects of testosterone replacement for the nonmotor symptoms of Parkinson disease. Arch Neurol 2002;59:1750-3

3 Tenover JS. Androgen administration to aging men. Endocrinol Metab Clin North Am 1994;23:877-92.

4 Gibb WR. Functional neuropathology in Parkinson's disease. Eur Neurol 1997;38:21-5

5 Craig AH, Cummings JL, Fairbanks L, et al. Cerebral blood flow correlates of apathy in Alzheimer's disease. Arch Neurol 1996;53:1116-20. 
6 Pluck GC, Brown RG. Apathy in Parkinson's disease. J Neurol Neurosurg Psychiatry 2002;73:636-42.

7 Isella V, Melzi P, Grimaldi M, et al. Clinical, neuropsychological, and morphometric correlates of apathy in Parkinson's disease. Move Disord 2002;2:366-71

8 Ringman JM, Diaz-Olavarrieta C, Rodriguez Y, et al. The prevalence and correlates of neuropsychiatric symptoms in a population with Parkinson's disease in Mexico. Neuropsychiatry Neuropsychol Behav Neurol 2002;15:99-105.

9 Aarsland D, Larsen JP, Lim NG, et al. Range of neuropsychiatric disturbances in patients with Parkinson's disease. I Neurol Neurosurg Psychiatry 1999;67:492-6

10 Starkstein SE, Mayberg HS, Preziosi TJ, et al. Reliability, validity and clinical correlates of apathy in Parkinson's disease. J Neuropsychiatry Clin Neurosci 1992:4:134-9.

11 Bimonte-Nelson HA, Singleton RS, Nelson ME, et al. Testosterone, but not nonaromatizable dihydrotestosterone, improves working memory and alters nerve growth factor levels in aged male rats. Exp Neurol 2003;181:301-12.

12 Janowsky JS, Chavez B, Orwoll E. Sex steroids modify working memory. J Cogn Neurosci 2000; 12:407-12.

13 Association AP. Diagnostic and statistical manual of mental diseases, 4th ed. Washington DC: American Psychiatric Association, 1994.
14 Grace J, Malloy PF. Frontal systems behavior scale. Odessa, FL: Psychological Assessment Resources, 2002.

15 Ready RE, OHt BR, Grace J, et al. Apathy and executive dysfunction in mild cognitive impairment and Alzheimer's disease. Am J Geriatr Psychiatry 2003;11:222-8.

16 Beck AT, Steer RA, Brown GK. Beck depression inventory - second edition. San Antonio, TX: The Psychological Corporation, 1996.

17 Diagnostics Q. Testosterone, free, bioavailable and total, 2003.

18 Hoehn MM, Yahr MD. Parkinsonism: onset, progression, and mortality. Neurology 1967; 17:427-42.

19 Harman S, Metter EJ, Tobin JD, et al. Longitudinal effects of aging on serum total and free testosterone levels in healthy men. J Clin Endocrinol Metab 2001;86:724-31.

20 Morley JE, Charlton E, Patrick P, et al. Validation of a screening questionnaire for androgen deficiency in aging males. Metabolism 2000;49:1239-42.

21 Howell S, Shalet S. Testosterone deficiency and replacement. Horm Res 2001;56(suppl 1):86-92.

22 Azad N, Pitale S, Barnes WE, et al. Testosterone treatment enhances regional brain perfusion in hypogonadal men. J Clin Endocrinol Metab 2003;88:3064-8.

23 Aging Nlo. Temporary NIA policy on funding human testosterone intervention studies, 2003

\section{Making Health Care Safer 2004}

21-22 October 2004

Royal College of Physicians, London

A two day conference for all professionals dedicated to providing safer health care for all.

Register now! Early booking discount available.

See website for details: www.quality.bmipg.com 